\title{
Supporting 3D Window Manipulation with a Yawing Mouse
}

\author{
Rodrigo Almeida and Pierre Cubaud \\ \{rodrigo.almeida, cubaud\}@cnam.fr \\ Centre d'Etudes et de Recherche en Informatique (CEDRIC) \\ Conservatoire National des Arts et Métiers (CNAM) \\ 292, rue Saint-Martin, 75003 Paris, France
}

\begin{abstract}
We present an interaction technique based on a yawing mouse (a device that senses the yaw orientation), designed for integral manipulation of 3D desktop windows in a three degrees-of-freedom space. We describe the construction of a prototype. A pilot study is conducted in order to investigate the performance gain expected with the yawing mouse. We then discuss some aspects of the form factors of devices intended to this kind of task.
\end{abstract}

\section{Author Keywords}

3D Interaction, input devices, multiple degree-of-freedom, mouse, usability study, integral manipulation.

\section{ACM Classification Keywords}

H5.2. Information interfaces and presentation.

\section{INTRODUCTION}

One of the trends of today's desktop interfaces in computer industry, supported by the omnipresence of accelerated graphic cards, is the integration of $3 \mathrm{D}$ metaphors and technology into conventional 2D paradigm [12]. A number of research works were also made in this direction $[2,3,11$, 13]. However, we believe that these new interfaces must be addressed not only by new metaphors, but also by interaction techniques yielding simple and efficient appropriation.

In this paper, we focus on the 3D desktop window metaphor. One of the goals of these interfaces is to enhance the screen-space use by supporting the visualization of multiple windows at the same time, proposing therefore an alternative to the overlapping-window model. Among the 3D approaches, little attention has been devoted to interaction techniques that handle intuitive and granular orientation - one of the key tasks for taking advantage of the screen space. Instead, arbitrary mappings, increasing

Permission to make digital or hard copies of all or part of this work for personal or classroom use is granted without fee provided that copies are not made or distributed for profit or commercial advantage, and that copies bear this notice and the full citation on the first page. To copy otherwise, to republish, to post on servers or to redistribute to lists, requires prior specific permission and/or a fee.

NordiCHI 2006: Changing Roles, 14-18 October 2006, Oslo, Norway

Copyright 2006 ACM ISBN 1-59593-325-5/06/0010 ..\$5.00 user's cognitive load and handling separately a rather integral task [7] (where translation and rotation can be performed naturally and simultaneously [14]) are adopted.

This paper presents a three degrees-of-freedom (DOF) interaction technique based on a yawing mouse (i.e., a mouse that senses rotation along its vertical axis). We assessed this technique in a 3D window manipulation task where the window is constrained to the "ground", movable along the left-right and near-far axis, and orientable along the vertical axis. The yawing mouse controls granularly, integrally, and through a natural mapping the three parameters of a window (two planar coordinates and the yaw angle).

We first introduce some related works on yawing devices and describe the yawing mouse prototype. Then, we report the evaluation that was performed and discuss its results. We conclude by presenting some considerations about our experimental design and directions for future works.

\section{A YAWING MOUSE}

\section{Related Works}

Many devices have been suggested for extending the mouse to multiple DOF. However, the standard, mass-product, mouse has itself evolved a lot in the last decade, mainly through vision-based technologies for capturing mouse moves, along with wireless connection to the host computer. Some technological aspects of the works referenced below are therefore out-of-date, but many interesting methodological arguments remain.

A 3DOF device, the Two-ball mouse [8] has been designed specifically for the capture of the yaw angle. As the name suggests, the device relies on ball-based mice mechanically coupled inside the chassis of a standard mouse. No formal performance is described in this exploratory paper.

The Rockin'Mouse [1] is a 4DOF device. Although it does not sense the yaw angle), it senses relative standard $\langle\mathrm{X}, \mathrm{Y}\rangle$ position and tilting along both axes. A formal evaluation has shown that at least a $30 \%$ performance gain over the regular mouse could be expected for 3D positioning tasks. In these experiments, only translations in the 3D space were investigated, but their experimental methodology and conclusions are relevant for our study. 


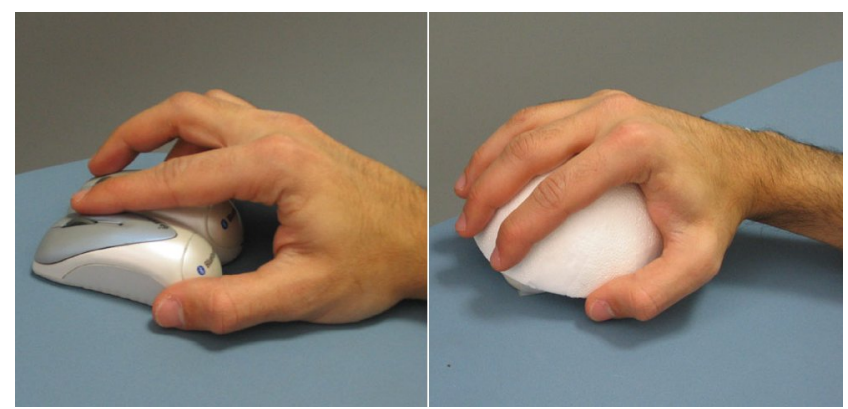

Figure 1: The 3DM (left) and the 3DR (right). Built with 2 mini bluetooth 800 DPI optical mice (IOGear-GME225B); each mouse measuring $3.2 \mathrm{~cm}$ (width), $8.5 \mathrm{~cm}$ (length), and $3 \mathrm{~cm}$ (height). The polystyrene hemisphere, housing the 3DR device had a $12 \mathrm{~cm}$ diameter and an $8 \mathrm{~cm}$ height.

The Videomouse [6] is a $6 \mathrm{DOF}$ device that uses a $\mathrm{CCD}$ camera for sensing its movements parallel to the surface (translation and orientation), and a certain degree of elevation over the surface. In order for these movements to be correctly and rapidly interpreted, the Videomouse requires a special surface with a printed pattern. The Videomouse (and the Two-ball mouse) may need to use either a clutching or a rate control mechanism for handling rotation.

The Toolstone [10] is a rectangular box-shaped 6DOF device that senses translation, orientation and tilting. The prototype box houses three Wacom magnetic trackers and hence, must also be used on a Wacom tablet. The Toolstone device was developed for more general applications than $3 \mathrm{D}$ interaction.

The more recent 3Style Mouse [4] is a cordless 3DOF mouse. The absolute yaw angle is provided by "two linear magnetic solid state sensors mounted at 90 degrees to each other" that act as a compass. It seems to regroup most of the features that we consider important for a yawing mouse, namely: being cordless, conveying no orientation through touch, and having an absolute orientation. However, we are not aware of any formal study of the 3Style Mouse performance.

\section{Prototype Implementation}

A simpler prototype than the Two-ball mouse can be built using two (recent) cordless laptop mice, glued together on their lateral side (figure 1). We used XInput, an extension library of X11, to obtain separately the coordinates of each mouse. When operating, the mice deliver four relative coordinates: $\left\langle\mathrm{X}_{1}, \mathrm{Y}_{1}\right\rangle$ for the left-side mouse, $\left\langle\mathrm{X}_{2}, \mathrm{Y}_{2}\right\rangle$ for the right-side one. If all four coordinates are initialized at 0 , a rotation is detected when the values of $Y_{1}$ and $Y_{2}$ mismatch. When the device is turned clockwise, $Y_{2}$ increases and $Y_{1}$ decreases symmetrically. The difference between $Y_{1}$ and $Y_{m}$, the mean $Y$ value (which is not in practice always null), is proportional to the yaw angle $\alpha$. The proportionality coefficient $\mathrm{K}$ will be defined, among other factors, by the distance separating the center of the optical sensor of the left mouse from that of the right

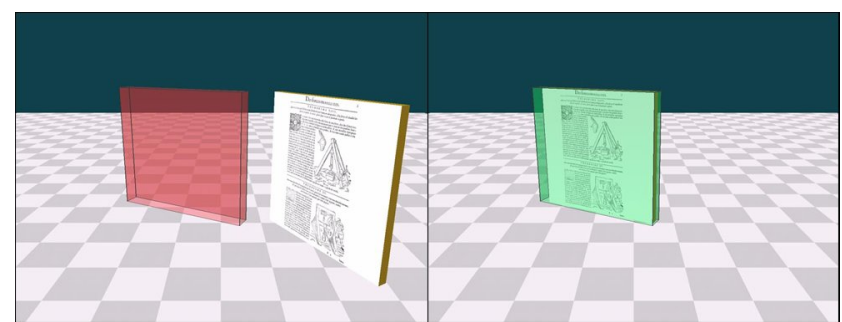

Figure 2: Screenshots before (left) and after (right) placing the box inside the target. The 3D scene was displayed on a 19 inches, $1280 \times 1024$ screen.

mouse. Thus, the relative position of the prototype is defined by $\left\langle\mathrm{X}_{\mathrm{m}}, \mathrm{Y}_{\mathrm{m}}\right\rangle$, and its relative orientation is defined by: $\alpha=\mathrm{K}\left(\mathrm{Y}_{1}-\mathrm{Y}_{\mathrm{m}}\right)$. The fact that only relative orientation can be obtained with such a device is not mentioned in [8].

For instance, if the yawing mouse is turned 90 degrees clockwise, and then moved to the left, its sensors will detect a movement along the $\mathrm{Y}$ axis, as it were brought closer to the user, and not along the $\mathrm{X}$ axis to the left. To circumvent this problem, we define atot, a variable to which each measure of $\alpha$ is added iteratively.

The "absolute coordinates" of the device $\left\langle\mathrm{X}_{\mathrm{a}}, \mathrm{Y}_{\mathrm{a}}\right\rangle$ are then derived as follow:

$$
\begin{aligned}
& X_{a}=\sin (\alpha t o t) X_{m}+\cos (\alpha t o t) Y_{m} \\
& Y_{a}=\cos (\alpha t o t) X_{m}-\sin (\alpha t o t) Y_{m}
\end{aligned}
$$

The accuracy of the device is clearly limited. Over time, the difference of $Y_{1}$ and $Y_{2}$ absolute values yields an accumulation of errors in the computation of atot. The prototype is however reliable enough for a study of the potential of yawing mice.

\section{PILOT STUDY}

We based our experiment design on the work reported in [5]. Sixteen volunteers (eleven male, five female, righthanded, mean age 27.6 years, mostly students at the University) accepted to participate. Our task scenario proposed a simplified version of a 3DOF window interface, i.e., using only one window. Our main goal was to compare the yawing-mouse based technique with conventional mouse based ones. We also looked for differences between two yawing mouse configurations (having distinct physical form factors).

The participants had to perform a 3DOF matching task, placing a rectangular solid box inside a target rectangular translucent box (figure 2). The solid box was yellow and had a black-and-white document facsimile applied as a texture onto one of its front faces. Both boxes were constrained to the ground and had the same height. The target box was 5\% larger, in width and in depth, than the controlled box. The solid box was considered to be matched with the translucent box only if it was oriented in such a way that its front face was visible to the participant. The translucent box, initially red, became green once the solid 
box was completely inside it, and correctly oriented. Translation movements, with both the yawing mouse and the regular mouse, were accomplished through a direct mapping between the device and the solid box, e.g., moving the device to the left, moved the box to the left. The way to handle the rotation of the solid box was the main difference among the experimental conditions.

Our four experimental conditions were: 3DM - the yawing mouse without housing; 3DR - the yawing mouse housed inside a polystyrene hemisphere (figure 1); $2 \mathrm{DW}-\mathrm{a}$ technique using the mouse's scroll-wheel for performing rotation; 2DM - a technique that, while mouse's left-button remained pressed, left-right mouse movement rotated the box. Rotating the 3DM, or the 3DR, rotated the box through a 1:1 control display mapping. The 2DW and 2DM used a regular tethered mouse. A unique set of 20 positions and orientations of both the target and the controlled box were previously randomly generated and stored in a static list. The 20 trials (a trial corresponded to place the solid box inside the translucent box) had, therefore, identical initial configurations for all 16 participants and for all four interaction techniques. The participants had, before the trial sequence of each interaction technique, a short demonstration about how it worked. They then started the trials, but the first five trials of each technique were a practice block; these trials were recorded, but ignored for the analysis. In summary, every one of the 16 participants tested all four experimental conditions, performing 15 valid trials under each one of the four conditions (960 valid trials). We used a within-subjects latin-square design (with four experimental-condition sequences) in order to control learning effects and differences among participants. The dependent variable was time to completion. Pressing the space bar both started and finished the trial (once the controlled box was completely inside the translucent one).

Our first hypothesis was that the yawing mouse based techniques should take advantage of their 3DOF integral control and outperform 2DM and 2DW. The second hypothesis is that the $3 \mathrm{DM}$, due to its asymmetric shape (sometimes conveying an orientation mismatching that of the window under control), would be worse evaluated by the participants than the 3DR.

\section{Results and Discussion}

The yawing mouse (3DM and 3DR) conditions presented more efficient results for about $70 \%$ of the trials (table 1). Figure 3 shows that the 3DR and (above all) the 3DM trials predominated in reduced completion times (2-4 seconds), while the 2DW and (above all) the 2DM trials predominated in longer completion times (5-8 seconds). The great variability of the measures suggests however that the differences between the 3DM and the 3DR conditions are not significant. This is confirmed by a KolmogorovSmirnov (KS) test over the four couples of distributions.

After the test we asked the participants to rank the four interaction techniques in an interval scale ranging from one

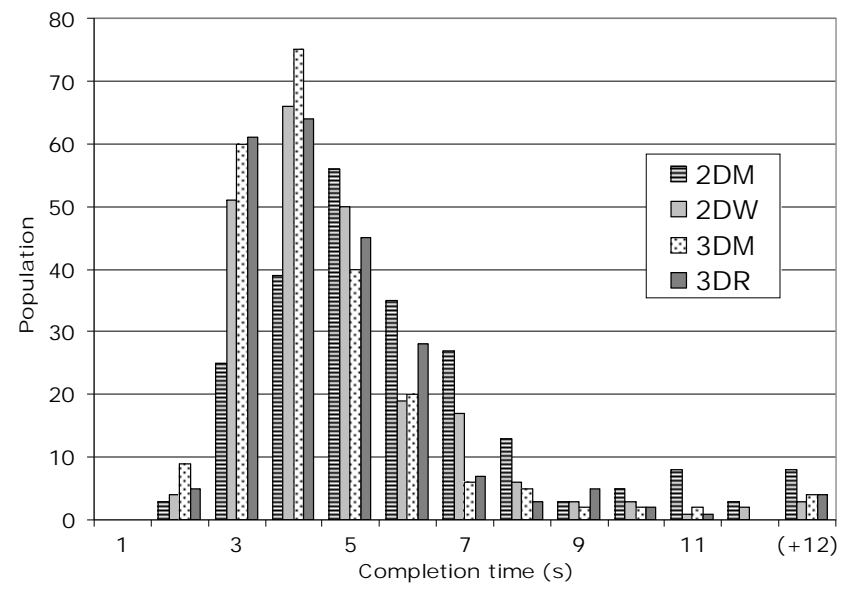

Figure 3: Histograms of completion time achieved by the participants using the 4 interaction techniques.

to five according to their preferences. Participants' preferences were well distributed among the four techniques, with a slight advantage to the yawing-mouse based ones. The 3DR technique had the best mean score (3.18) while the 2DW technique had the worst (2.68); the $3 \mathrm{DM}$ and the 2DM had similar means (2.93 and 3.00 respectively). We may interpret these results optimistically since the yawing-mouse based techniques, using devices until then unknown to the participants, were slightly better ranked than the mouse-based ones.

\begin{tabular}{|r|c|c|c|c|}
\cline { 2 - 5 } \multicolumn{1}{c|}{} & 2DM & 2DW & 3DM & 3DR \\
\hline Mean (s) & 5.0 & 4.2 & 3.6 & 3.8 \\
\hline Std. Dev. (s.) & 2.0 & 1.6 & 1.3 & 1.5 \\
\hline is Fastest (\%trials) & 8.4 & 21.3 & 37.7 & 32.4 \\
\hline
\end{tabular}

Table 1 : Results for task completion time using the four interaction techniques.

Our results confirm the first hypothesis, that yawingmouse techniques outperform the mouse-based ones in the defined 3DOF task. Concerning the second hypothesis, although 3DR having been slightly better evaluated than the 3DM, the results are not conclusive.

3DR and 3DM were considered "more natural" by some participants, probably due to the direct mapping of the three degrees-of-freedom. Using 3DR and 3DM, participants were never confused (neither had to learn) about which the sense of the rotation was (as happened many times under 2DM and 2DW). Compared to the mouse-based techniques, 3DR and 3DM seemed very efficient, since the former required sometimes long sequence of alternations between rotation and translation to match the final angle. This "twosteps procedure" of the 2DW and 2DM was criticized by some participants. In addition, the 2DW seemed inefficient for large angle rotations. The 3DM and 3DR presented an efficient tradeoff between speed and precision for windoworientation handling - participants performed fast rotations and, approaching the correct angle, naturally slowed down 
to a precision rotation. Since the 3DM device was smaller, participants grasped it with their finger tips; however, due to its asymmetric shape, this "precision grip" [9] was not sufficient to yield an easy rotation handling as the one found in [15]. On the other hand, this asymmetry provided tactile cues that seemed to ease orientation. This may explain why participants tended to make one large (and awkward) rotation to roughly reach the final orientation and, then, a second smaller movement to match the correct angle. Concerning the 3DR, participants laid their entire hands on the device surface. During rotation, they abandoned this grasp and used the finger tips, trying to grip either the side (more efficient) or the top of the device (more awkward). We observed that participants reached the matching orientation through many quick, short, and iterative rotations. For many users, however, the device was too large and, therefore, hard to grasp.

In summary, affording "whole hand grasp", as conventional mice do, may be problematic for yawing devices since they require large muscle group which may lead to inefficient and straining movements. Smaller devices, affording fingertip grasp are a best hint for quick and precise manipulations. In addition, a symmetric shape may better support these small finger-movements yielding efficient rotation handling [15].

\section{CONCLUSION AND FUTURE WORKS}

3D-window interfaces need an input device to let them as usable as the mouse did for 2D/WIMP interfaces. We presented a specific approach: an interaction technique, based on a yawing mouse, conceived to manipulate 3D windows in a $3 \mathrm{DOF}$ space. The results of our evaluation are positive and foster further investigations. Compared with the mouse techniques, however, the performance gain was small. We believe that more complex tasks (e.g., manipulation of multiple windows), imposing a larger duration to the task, may produce clearer results (but also more variables to control). In future works, we aim to conduct a more ambitious empirical study in order to investigate aspects seeming relevant: correlation between the performance of a technique and the length of the rotated angle; the simultaneity of translation and rotation using 3DOF integral devices; and detailed explorations of some of the device form factors. In addition, we plan to explore using of the yawing mouse in different interaction contexts, such as video and audio skimming, and interfaces that mimic potentiometers. Our prototype can be easily built, and we encourage those who want to replicate the experiment, or to try different configurations, to do it.

\section{ACKNOWLEDGMENTS}

We thank Patrick Baudisch for the insightful discussion and references, Eduardo Almeida for the help with the data analysis, Alexandre Topol and Jérôme Dupire for their comments, the anonymous reviewers of previous versions of this paper for their useful suggestions, and all the colleagues who participated in the experiment.

\section{REFERENCES}

1. Balakrishnan, R., Baudel, T., Kurtenbach, G., and Fitzmaurice, G. The Rockin'Mouse: Integral 3D Manipulation on a Plane. Proc CHI 1997, ACM Press (1997), 311-318.

2. Card, S., Robertson, G., and York, W. The WebBook and the Web Forager: An information workspace for the World-Wide Web. Proc. CHI 1996, ACM Press(1996), 111-117.

3. Chapuis, O. and Roussel, N. Metisse is not a $3 \mathrm{D}$ Desktop. Proc. UIST 2005, ACM Press (2005), 13-22.

4. Cylo 3Style Mouse Page. http://www.hifidesign.com.au/products.html/.

5. Hinckley, K., Tullio, J., Pausch, R., Proffitt, D., and Kassell, N. Usability Analysis of 3D Rotation Techniques. Proc. UIST 1997, ACM Press (1997), 1-10.

6. Hinckley, K., Sinclair, M., Hanson, E.,.Szeliski, R., and Conway, M. The VideoMouse: a camera-based multidegree-of-freedom input device. Proc. UIST 1999, ACM Press (1999), 103-112.

7. Jacob, R. and Sibert, L. The Perceptual Structure of Multidimensional Input Device Selection. Proc. CHI 1992, ACM Press (1992), 211-218.

8. MacKenzie, I., Soukoreff, R., and Pat, C. A two-ball mouse affords three degrees of freedom. Extended Abstracts of CHI 1997, ACM Press (1997), 303-304.

9. MacKenzie, C. and Iberall, T. The Grasping Hand. North-Holland, Amsterdam, 1994.

10. Rekimoto, J. and Sciammarella, E. ToolStone: effective use of the physical manipulation vocabularies of input devices. Proc. UIST 2000, ACM Press (2000), 109-117.

11. Robertson, G., van Dantzich, M., Robbins, D., Czerwinski, M., Hinckley, K., Risden, K., Thiel, D., and Gorokhovsky, V. The task gallery: a 3D window manager. Proc. CHI 2000, ACM Press (2000), 494-501.

12. Sun Project Looking Glass. http://www.sun.com/software/looking_glass/.

13. Topol, A. Immersion of Xwindow applications into a 3D workbench. Extended Abstracts CHI 2000, ACM Press (2000), 355-356.

14. Wang, Y., MacKenzie, C.L., Summers, V.A., and Booth, K.S. The structure of object transportation and orientation in human-computer interaction. Proc. CHI 1998, ACM Press (1998), 312-319.

15.Zhai, S., Milgram, P., and Buxton, W. The influence of muscle groups on performance of multiple degree-offreedom input. Proc. CHI 1996, ACM Press (1996), 308-315. 